ASLI QoL 2017
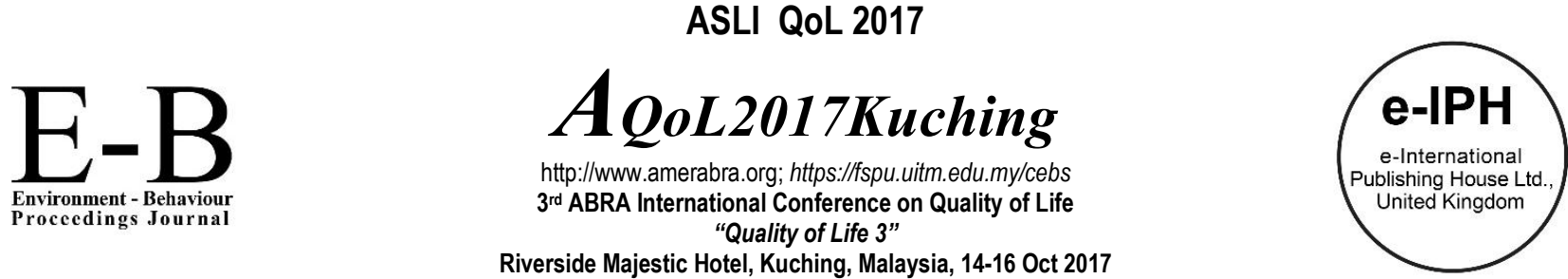

\title{
The Effect of Different Locations of Simulated Central Vision Scotoma on Quality of Reading
}

\author{
Noor Halilah Buari, Muhammad Zahir Nur-Zahirah \\ Optometry program, Faculty of Health Sciences, \\ Universiti Teknologi MARA Cawangan Selangor, 42300 Bandar Puncak Alam, Selangor, Malaysia \\ noorhalilah.buari@gmail.com \\ Tel:+60126121582
}

\begin{abstract}
As a person read, the eyes move to extract information from the printed text. Reading became disturbed when obstructed by the scotoma. The effect of reading and eye movement were investigated between different locations of simulated central vision scotoma. The time to read the text and the eye movements were recorded and tracked among eleven participants. The reading speed showed a significant difference at different locations of central scotoma simulation. The presence of central scotoma affects the quality of reading as the eye moved slowly during reading in order to comprehend the text.
\end{abstract}

Keywords: reading speed; simulated scotoma; central scotoma

eISSN: 2398-4287@ 2017. The Authors. Published for AMER ABRA by e-International Publishing House, Ltd., UK. This is an open access article under the CC BYNCND license (http://creativecommons.org/licenses/by-nc-nd/4.0/). Peer-review under responsibility of AMER (Association of Malaysian Environment-Behaviour Researchers), ABRA (Association of Behavioural Researchers on Asians) and cE-Bs (Centre for Environment-Behaviour Studies), Faculty of Architecture, Planning \& Surveying, Universiti Teknologi MARA, Malaysia.

https://doi.org/10.21834/e-bpj.v2i6.949

\subsection{Introduction}

Scotoma is defined as darkness or blind area within one's field of view and it varies in size and shape while, the surrounding area is a normal vision (Kelley, 1983). Scotoma is classified into two categories, which are central and peripheral scotoma. The scotoma varies in size and shape and had different effect towards activity of daily living such as in reading (Crossland et al., 2004; Fine \& Rubin, 1999; Sunness et al., 1996). Seiple (2013), states that central scotoma usually related to diseases such as age-related macular degeneration (AMD) and diabetic retinopathy (Seiple et al., 2013). A person with central scotoma would have difficulty to recognize faces and unable to see printed text even for the large size such as newspaper headlines (Brown et al., 2002).

Eye movement is a key element in the process of reading and extracting information from an object or target. During reading, the eyes make a saccadic movement which the rapid eye movement that continuously move while the eyes look for something or move from one object to another object (Rayner, 1998). Fixation happens during the stable state of the eye in between the saccades eye movements. The fixation lasts about 200-300ms (Rayner, 2009a). At this moment, the information processing take part since the object falls within the fovea. Regression is an eye movement involved during reading. Regression happens when the eye moves back to the previous letter or word during reading (Rayner \& Fischer, 1996) This can be due to the linguistic problem, oculomotor problem or text difficulty (Rayner \& Fischer, 1996). Patient with central scotoma frequently experience reading difficulty that can be attributed to reduce acuity, increase crowding or unstable fixation that associate with central visual field disturbance (Falkenberg et al., 2007). Study done by Bernard et al. showed that the larger size of scotoma, the slower the reading speed (Bernard et al., 2007). A similar result was showed in reading rates that were slower with scotoma and cataract at threshold letter size compared with normal vision

eISSN: 2398-42870 2017. The Authors. Published for AMER ABRA by e-International Publishing House, Ltd., UK. This is an open access article under the CC BYNCND license (http://creativecommons.org/licenses/by-nc-nd/4.0). Peer-review under responsibility of AMER (Association of Malaysian Environment-Behaviour Researchers), ABRA (Association of Behavioural Researchers on Asians) and cE-Bs (Centre for Environment-Behaviour Studies), Faculty of Architecture, Planning \& Surveying, Universiti Teknologi MARA, Malaysia.

https://doi.org/10.21834/e-bpj.v2i6.949 
(Fine \& Rubin, 1999b).

As the center field of view blocked, the reading rate declines and eye movement patterns changed (Fine \& Rubin, 1999b). Previous studies showed there was a significant correlation between the sizes of absolute scotoma with reading speed and reading acuity but not with relative scotoma (Ergun et al., 2003). Absolute scotoma caused disturbance in reading rate and changed the pattern of fixation (Sunness et al., 1999). The eyes changed the fixation pattern outside scotoma area, mostly towards the right visual field space than towards the superior and left visual field space. The reading speed was highly correlated with the size of scotoma. The reading speed faster with the scotoma above the fixation and became slower with the scotoma to the left (Sunness et al., 1999). Central scotoma can be happened either on one eye or both eyes. In bilateral age-related macular degeneration, viewing through both eyes gave an advantage to increase the reading speed than with monocular viewing (Kabanarou \& Rubin, 2006). However the pattern of the eye movement was similar in either monocular or binocular viewing. The visual field defect had delayed saccades eye movement which was lower in the frequency of express saccades compared to a normal person (Kanjee et al., 2012). Similarly, the mean saccade latencies were longer in moderate visual field defect and pre-perimetric visual field defect than in the normal group either with static and moving target (Lamirel et al., 2014). Less fixation stability was occurred when looking at peri-central target among those who affected with central scotoma (Bellmann et al., 2004).

Several simulated scotoma studies have been done to investigate the eye movement patterns in various aspects. It was found that the reading speed became slower in simulated vision impaired with central scotoma than in normal vision (Fine \& Rubin, 1999b). When the eye is blurred and obstructed with scotoma, the saccadic eye movement became shorter, number of saccades and fixation duration were increased. The eye needs double the time to search an object when simulated with central scotoma (Bertera, 1988). Fixation duration also increased by $15 \%$ as compared to the normal vision condition. Simulation central scotoma caused the eye took longer time to identify the word and made more fixations (Fine \& Rubin, 1999a). The reading speed was slower when attending to left of visual field with simulated scotoma than to the right of visual field. The eyes made more regression and smaller saccades when attending to the left. On the other hand, the subjects who have been simulated with central horizontal scotoma showed high preference to use inferior visual field than the superior visual field (Varsori et al., 2004). Changes in the eye movement patterns could be due to the loss of sensory and oculomotor disturbance due to the simulation scotoma. However, the eye could easily adapted with it and opted eye viewing strategy to improve the quality and performance of reading.

It was suggested and proved that the reading and the eye movement were significantly affected at some extent and location of the central visual field defect. The scotoma could affect one's quality of reading and changed the pattern of eye movements. The reading difficulty was a common daily activity that reported among those with central scotoma who seek for vision rehabilitation. Improving reading quality is one of the challenges faced by the vision practitioner, which nowadays reading is not only about reading book but wider scope from that. Various studies were done to investigate the reading quality and eye movement pattern either in real or simulated central scotoma conditions. However, the location of the central scotoma relative to the fovea was quite limited in published articles. Simulation study was very important to predict the real condition by controlling several aspects during the experiment. It could be a pilot study before investigate on the real patient. The characteristics of the eye movement during reading and investigation on the location of visual field defect would play a role in predicting the quality of reading. Thus, a study investigating the reading and eye movement patterns at the different location of central scotoma, which has been simulated was proposed in this article.

\subsection{Materials and Methods}

A comparative study was conducted to investigate the effect of reading speed and eye movement patterns in four simulated conditions, which were at nasal, temporal, superior and inferior of the fovea. The simulation was made using a goggle. The size and shape of simulation central scotoma were derived from the previous study (Varsori et al., 2004). The central scotoma was a circular disc subtended about $1.5^{\circ}$ of visual angle. Based on the calculation, the visual angle was approximately $10 \mathrm{~mm}$ height at $40 \mathrm{~cm}$ reading distance. The fig. 1 showed the central scotoma simulation.

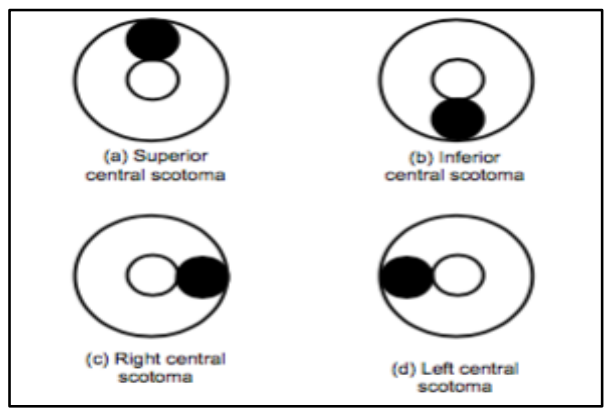

Fig. 1: The illustration of the location of central scotoma simulation on the goggle.

Seven Malay-language reading texts were used as reading stimuli in this study. The texts contained 1 paragraph, $4-5$ lines and 50 words. It was extracted from the Malay language textbook used by the primary school under Malaysian Ministry of Education. Since the experiment consisted of 5 conditions, i.e normal vision condition, central scotoma simulation at superior, inferior, right and left, 5 texts were used. One text was used as demonstration text prior to the actual experiment, while another one text was used as a backup 
text. The size of text was N8 print size or equivalent to 0.4 LogMAR, which represent the newspaper print size. The texts were printed on the matt surface white paper with high contrast using LaserJet printer.

Three-D Video Oculography (3D VOG) was used to track and quantify the horizontal, vertical and torsional eye movements. The 3D VOG is fully non-invasive video image processing technology, using the head-mounted infrared video camera (VOG mask). Two video cameras are mounted on the mask. For each camera, the infrared LED sources are provided to illuminate each eye. All required components of the system i.e the goggle and infrared video camera are combined with a high-performance computer workstation. The real-image processing, calibration, stimulus-software interface, built-in data analysis, and data recording are integrated within an easyto-use MS Windows application version 5.04.02. The screen resolution is set to $1024 \times 768$ or greater with 32-bit colour depth or greater. Fig. 2 showed the setting of 3D Video Oculography on the patient.

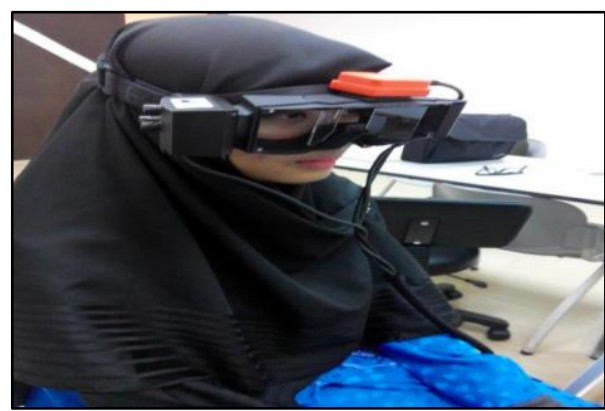

Fig. 2: The setting of the experiment using the 3-D video oculography. The goggle was set on the participant head with the infrared

The sample size was calculated using $G$ power 3.1.9.2 based on ocular movement from previous findings (Cerulli et al., 2014). The alpha error of probability was set at 0.05 ; the effect size (d) was 0.98 . With the power of study at 0.85 , a number of participants needed in this study were sixteen. However, only eleven participants' data can be analyzed in this study. Five data were missing and corrupted when the instrument breakdown in the middle process of data analysis.

Eleven young adults aged 23 to 26 years old participated. The participants should have no remarkable history of ocular disease, corrected visual acuity same or better than $6 / 6$. Those who had a vision correction, it should be less than or equal with -2.00 diopter sphere and/or -0.50 diopter cylinder. The participants also had no gross binocular vision problems. The young adults participants with a good visual status were chosen to avoid confounding factors in this study. This experiment was intended solely to investigate at the effect of central scotoma on reading without other ocular disorders. The presbyopia candidates aged more than 40 years old were excluded due to the difficulty to correct the near refractive error (spectacle correction) while fitted with the 3D-VOG goggle. The participants need to be fluently read the Malay language material. Inform consent was obtained prior to the experiment procedures and this study adheres to the tenets of the declaration of Helsinki and the institutional review board approved this study (Approval no. 600-FSK/PT.5/2).

Vision screening was done prior to the reading and eye movement measurements. Distance visual acuity was measured using Bailey-Lovie LogMAR chart at 3 meters distance. The subjective refraction was performed to ensure the refractive error within the acceptable range. Those who had refractive errors was then corrected with Focus Dailies soft contact lenses (Material: nelfilcon A, water content: $69 \%$ ) with base curve $8.6 \mathrm{~mm}$ and diameter of $13.8 \mathrm{~mm}$. Participants who were fitted with soft contact lenses were screened their ocular health and tears status using a slit lamp biomicroscope. The tears status was determined using the tear break up time (TBUT) method. The cornea was dyed with fluorescein strips, after several blinks, the participants were asked to hold blink. The first "dry spot" which remarks with the black spot on the cornea was observed through the slit lamp biomicroscope with cobalt blue filter. The time taken from the participants' holds their last blink to the first 'dry spot' was recorded as the tear break up time in seconds. Acceptable TBUT was more or equal to 8 seconds (Jamaliah \& Fathilah, 2002). Gross binocular vision problem was screened with remote near point of convergence and near point of accommodation using the Royal Air Force (RAF) rule. The remote near point of convergence (NPC) and near point of accommodation (NPA) were measured with the push-up and push down methods. The break and recovery points were recorded for NPC while blur, break and recovery points were noted for NPA measurement. Recording of NPC and NPA were in centimeter. Both NPC and NPA should be within norms values of young adults (Rosenfield \& Cohen, 1996; Scheiman et al., 2003).

The eye movements were then recorded using 3D- VOG while reading the Malay texts. The simulation was attached on the 3-D VOG goggle on both of participants eyes. The simulations and normal vision condition were chosen at random sequence for each participant. The calibration procedure was conducted before the actual measurements to capture the eye position and set as a reference position. It was performed as the eye and head straight ahead looked at the given target on the screen monitor. The pupil of the eyes was monitored and should always at the center of the camera live image. Then the participants were asked to read the Malay text that placed on the slanted reading table at 45 degrees with $40 \mathrm{~cm}$ distance from the eye plane. The reading should be a loud, clear pronunciation at normal speed. Any error made such as mispronunciation, substitution, reversal, refusal, omission and addition was noted as reading errors (Khalid et al., 2017). The reading was recorded with audiotape for post-experiment evaluation of reading time and reading error. The reading speed was quantified as numbers of correct word that can be read in a minute (words per minute). Once the participants ready, they were instructed to start reading the text and at the same time, the eye movement measurement was started. The torsional test was used to record the eye movement. It was depended on the visibility of the pupil. The participants were 
always reminded to keep the eyes widely open. The eye position during the torsional eye movement measurement was at the range of $\pm 20^{\circ}$ horizontally and $\pm 20^{\circ}$ vertically. The eye movements were determined as numbers of fixation and numbers of regression. Fixation is the point that the eye stationary to process the visual output while the regression is the right-to-left eye movement backs the previous words to recapture the missing information during reading (Rayner, 1998).

Statistical analysis was performed using SPSS version 21.0. The descriptive data were presented as mean, median and standard deviation. The comparison of reading speed and the eye movement in term of numbers of fixation and numbers of regression between the normal condition and simulated scotoma conditions was done with one-way ANOVA. The significance level was set at $p<0.05$.

\subsection{Results}

The reading speed and the eye movement data were normally distributed using Shapiro-Wilk test $(p>0.05)$. Therefore, the analysis was carried out using parametric test, one-way ANOVA.

\subsection{Comparison of reading speed between normal condition and simulated central scotoma}

Reading speed between normal vision, superior, inferior, right and left scotoma simulation were $129 \pm 20$ wpm, 109 \pm 21 wpm, $102 \pm 12$ wpm, $113 \pm 20$ wpm and $115 \pm 16$ wpm, respectively as shown in Fig. 3 . The reading speed was reduced significantly with the simulated scotoma as compared with the normal condition, $[F(4,54)=3.17, p=0.02]$. The inferior simulated scotoma gave the slowest for the inferior scotoma stimulation as compared to the other conditions ranging from $85 \mathrm{wpm}$ to $128 \mathrm{wpm}$.

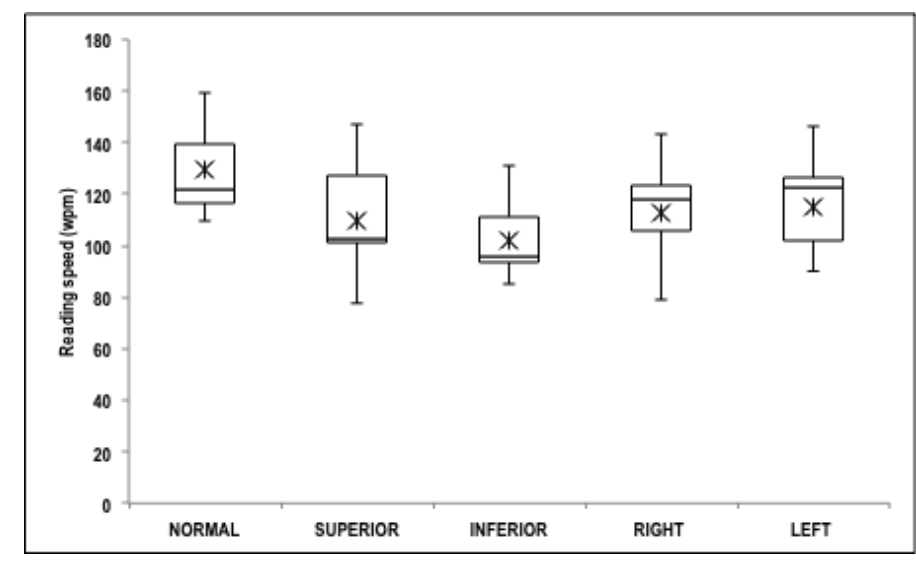

Fig. 3: The box and plot of reading speed on normal condition and in four simulated scotoma conditions. The error bar showed the maximum and minimum values of the reading speed for each condition. The lower edge of the box represents the 1st quartile and upper edge of the box represents the 3 rd quartile. The star marker $\left(^{*}\right)$ in the middle of the box showed the mean of reading speed.

\subsection{Comparison of eye movements between normal condition and simulated central scotoma}

The eye movements were measured based on numbers of fixation and numbers of regression. The comparison of number of fixation while reading was found not significantly difference $[F(4,54)=0.61, p=0.65]$ between the normal vision and simulated central scotoma either at the superior, inferior, right or left region of the fovea. The mean and standard deviation for numbers of fixation in the condition without stimulation was $19 \pm 7$. The mean numbers of fixation made during reading with the simulated central scotoma towards the superior, inferior, right and left region of fovea were $15 \pm 10,17 \pm 9,18 \pm 9$ and $14 \pm 6$, respectively. Reading with the simulated central scotoma at the right of the fovea showed the highest number of fixation with the range between 5 to 33 numbers of fixations. This indicated the eye with scotoma at the right region stop more during reading than in the other locations of scotoma. This was followed with inferior scotoma, superior scotoma and left scotoma. The mean of numbers of fixation was represented in Fig. 4.

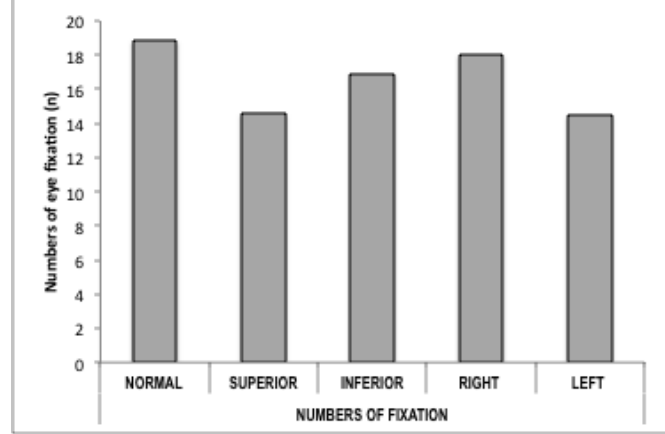

(a)

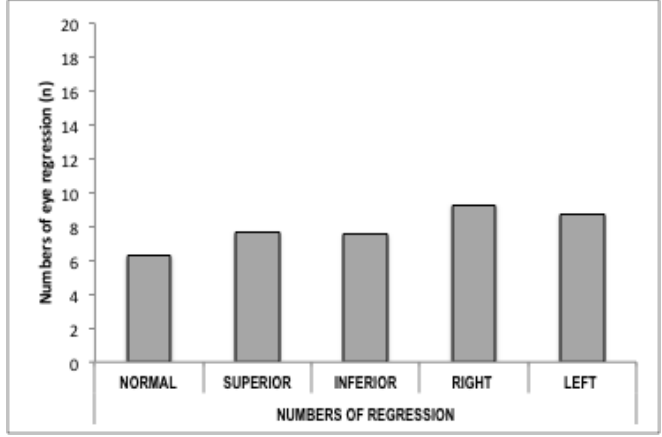

(b)

Fig. 4: (a) Comparison of numbers of fixation duration between normal condition and four simulated central scotoma (b) Comparison of numbers of regression duration between normal condition and four simulated central scotoma 
The numbers of regression made during reading for the normal condition was $6 \pm 3$ ranging between 1 to 13 numbers of regressions. When reading with simulated central scotoma at the superior, inferior, right and left region from the fovea, the eye made regression movement of $8 \pm 4,8 \pm 4,9 \pm 4$ and $9 \pm 5$., respectively. The regression movements were approximately similar between simulated central scotoma location, $F(4,54)=0.90, p=0.47$. However, the eyes made more regression movements when they were simulated with scotoma than in the normal condition. As compared to the numbers of fixation and numbers of regression, the eyes stop more when reading the text than regress back to the previous word during reading. This was shown in Fig. 4 that numbers of fixation was higher than the number of regression.

\subsection{Discussion}

The reading speed showed a significant reduction when reading with the eye that simulated with central scotoma regardless its locations. It might be due to the presence of artificial central scotoma that disturbs the reading process. Obstruction at the inferior region of the visual field showed the slowest reading speed as compared to the other locations of the scotoma. Previous study showed that reading speed was slower when it was simulated with a combination vision impaired and central scotoma than in normal vision (Fine \& Rubin, 1999b). The larger text size was needed to improve the quality of reading. Even with larger text size, it changed the pattern of the eye movement. In our study, the chosen text size was equivalent to the size of newspaper prints. Apart from the effect of the simulated central scotoma, the small text size could be the factor that affects the quality of reading among our participants. Other findings showed that the subjects with simulated central scotoma took a longer time to identify letters and words when attending to the left of visual field than to the right of visual field (Fine \& Rubin, 1999a). Reading speed with artificial central scotoma simulation was quite slower than normal condition because the participants may lose the use of fovea (Fine \& Rubin, 1999b). The loss of central visual field reduced the acuity and contrast sensitivity. Besides that, the participants with real scotoma usually adopted a preferred retinal locus which substitutes the used of fovea to the peripheral retinal region to the obtained clarity of vision (Fine \& Rubin, 1999b). Based on a study done by Pesudovs and colleagues, a highly significant difference was found in reading speed between six groups of ocular disorder conditions (Pesudov et al. , 2002). It was found that the cataract, central vision loss, central vision loss with cataract, peripheral vision loss, peripheral vision loss with cataract and also control group read at $93.1 \mathrm{wpm}, 29.2 \mathrm{wpm}, 30.0 \mathrm{wpm}, 85.0 \mathrm{wpm}$, $89.3 \mathrm{wpm}$ and $102.1 \mathrm{wpm}$, respectively. These findings clearly supported that the central vision loss displayed the lowest reading speed than other conditions. The size of absolute scotoma was correlated significantly with the reading capability and reading speed (Ergun et al., 2003). The reduction in reading speed in those with central scotoma was suggested influenced by the visual span which the size of the window that can be seen clearly at the fixation point (Cheong et al., 2009). The size of visual span in age-related macular degeneration (ARMD) was found substantially smaller than normal vision at the central field of view. The size of visual span contributed $36 \%$ of the variance in ARMD reading speed.

The number of fixation was not statistically significant in simulated central scotoma locations as compared with normal vision. But, the simulated central scotoma at right and inferior of fovea was the most that affected in this reading experiment. The eye tended to make more stop during reading with simulated central scotoma at the right of fovea. This could be happened due to the obstruction of view with the simulated scotoma on the next word to be read in the text. It was reported that the size of scotoma size influenced the number of fixation, fixation duration and searching time. The study investigated 3 sizes of scotoma, which were the absolute scotoma, relative scotoma at $0.23^{\circ}$ gap sizes and relative scotoma with $0.93^{\circ}$ gap sizes. The larger the size of scotoma, the more number of fixations was made (Cornelissen et al., 2005). The previous study showed that, the time taken for word identification was longer in simulated central scotoma and participants tends to make a higher number of fixations (Fine \& Rubin, 1999b). It was because the participants need to search the word in their visual space and search the optimal fixation location within the word to recognized and process word. The number of fixation was also found increased as the field of view became constricted (Cornelissen et al., 2005). The time taken to search a target or object was increased as the peripheral visual field defect constricted from $15^{\circ}$ to $10^{\circ}$ and $5^{\circ}$ of field of view. The stability of fixation was also found to be less in patients with central scotoma when looking at per-central region (Bellmann et al., 2004).

The numbers of regression were also not significantly different between the normal condition and simulated central scotoma conditions. However, the eyes made more regression movements when they were simulated with scotoma than in the normal condition. The regression movement happened as the eye moves towards backward to re-fixate on the previous letters or words that had been read. The adult reader was considered as experienced and skilled reader compared to the children. The probability of the re-fixation among the reading skilled readers was low during reading (Rayner, 2009a). Furthermore, as the reading skill increased, the number of regression was decreased (Rayner, 2009b). An individual made regression movement could be due to understanding problem or the oculomotor problem. Since the participants in our study were aged from 23 to 26 years old, the participants were categorized as the skilled reader. As hypothesized, the participants should not make higher regression movement. However, it happened differently. The higher numbers of regression in simulated central scotoma conditions than in normal condition could be happened due to oculomotor problem and understanding problem. As the scotoma obstructed the field of view during reading, the participants might have difficulty to choose the fixation point to the next word in the text. This could disturb the reading process to comprehend the text. Therefore, the eye tends to make more regression movement with the simulation central scotoma. 


\subsection{Conclusion}

The reading speed was reduced with central scotoma simulation but not in the eye movement patterns in term of numbers of fixation and numbers of regression. This could be due to the limited number of the participants in this study. However, this simulation study could give an insight on the prediction of reading and the eye movement patterns when applied on the real and actual central scotoma condition as in patient with age related macular degeneration. The trend of the eye movements with central scotoma simulation showed that the eye tended to stop more and made regression movements, which lead to the increment of time to finish reading the text. This might caused the reading speed became slow. The findings suggested that the obstruction of central field with simulated scotoma especially on the right region of fovea could affect the reading process as well as the quality of reading in one's daily life.

\section{Acknowledgement}

The authors gratefully acknowledge the Malaysian Ministry of Higher Education in providing the Fundamental Research Grant Scheme awarded by (600-RM1/FRGS 5/3 (119/2014)) research grant. A huge thanks was also dedicated to Prof Dr. Ai-Hong Chen and Shauqiah Jufri for enormous support and guidance.

\section{References}

Bellmann, C., Feely, M., Crossland, M. D., Kabanarou, S. a, \& Rubin, G. S. (2004). Fixation stability using central and pericentral fixation targets in patients with agerelated macular degeneration. Ophthalmology, 111(12), 2265-70. http://doi.org/10.1016/j.ophtha.2004.06.019

Bernard, J.-B., Anne-Catherine, S., \& Eric, C. (2007). Page mode reading with simulated scotomas: a modest effect of interline spacing on reading speed. Vision Research, 47(28), 3447-59. http://doi.org/10.1016/j.visres.2007.10.005

Bertera, J. H. (1988). The effect of simulated scotomas on visual search in normal subjects. Investigative Ophthalmology and Visual Science, 29(3), 470-475.

Brown, M. M., Brown, G. C., Sharma, S., Landy, J., \& Bakal, J. (2002). Quality of Life With Visual Acuity Loss From Diabetic Retinopathy and Age-Related Macular Degeneration. Archives of Ophthalmology, 120(4), 481-484. Retrieved from http://www.ncbi.nlm.nih.gov/pubmed/11934322

Cerulli, A., Cesareo, M., Ciuffoletti, E., Montanaro, M. L., Mancino, R., Mirisola, C., ... Cerulli, L. (2014). Evaluation of eye movements pattern during reading process in patients with glaucoma: a microperimeter study. Eur J Ophthalmol, 24(3), 358-363. http://doi.org/10.5301/ejo.5000397

Cheong, A. M. Y., Legge, G. E., Lawrence, M. G., Cheung, S. H., Ruff, M. A., \& Otr, L. (2009). Relationship between visual span and reading performance in AgeRelated Macular Degeneration. Vision Res, 48(4), 577-588.

Cornelissen, F. W., Bruin, K. J., \& Kooijman, A. C. (2005). The influence of artificial scotomas on eye movement during visual search. Optometry \& Vision Science 82(1), 27-35.

Crossland, M. D., Culham, L. E., \& Rubin, G. S. (2004). Fixation stability and reading speed in patients with newly developed macular disease. Ophthalmic \& Physiological Optics, 24(4), 327-33. http://doi.org/10.1111/j.1475-1313.2004.00213.x

Ergun, E., Maár, N., Radner, W., Barbazetto, I., Schmidt-Erfurth, U., \& Stur, M. (2003). Scotoma size and reading speed in patients with subfoveal occult choroidal neovascularization in age-related macular degeneration. Ophthalmology, 110(1), 65-69. http://doi.org/10.1016/S0161-6420(02)01566-X

Falkenberg, H. K., Rubin, G. S., \& Bex, P. J. (2007). Acuity, crowding, reading and fixation stability. Vision Research, 47(1), 126-135 http://doi.org/10.1016/j.visres.2006.09.014

Fine, E. M., \& Rubin, G. S. (1999a). Reading with simulated scotomas: Attending to the right is better than attending to the left. Vision Research, 39(5), $1039-1048$. http://doi.org/10.1016/S0042-6989(98)00208-9

Fine, E. M., \& Rubin, G. S. (1999b). The effects of simulated cataract on reading with normal vision and simulated central scotoma. Vision Research, 39(25), 42744285. http://doi.org/10.1016/S0042-6989(99)00132-7

Jamaliah, R., \& Fathilah, J. (2002). Prevalence of dry eye in University Malaya Medical Centre. Medical Journal of Malaysia, 57(4), 390-397.

Kabanarou, S. A., \& Rubin, G. S. (2006). Reading With Central Scotomas : Is There a Binocular Gain ? Optometry \& Vision Science, 83(11), 789-796.

Kanjee, R., Yucel, Y. H., Steinbach, M. J., González, E. G., \& Gupta, N. (2012). Delayed saccadic eye movements in glaucoma. Eye and Brain, 4, 63-68.

Kelley, J. (1983). Funduscopically controlled scotometry. Trans Am Ophthalmol Soc, 81, 592-629.

Khalid, N. M., Buari, N. H., \& Chen, A. (2017). Comparison of Oral Reading Errors between Contextual Sentences and Random Words among Schoolchildren. International Education Studies, 10(1), 47-55. http://doi.org/10.5539/ies.v10n1p47

Lamirel, C., Milea, D., Cochereau, I., Duong, M.-H., \& Lorenceau, J. (2014). Impaired saccadic eye movement in primary open-angle glaucoma. Journal of Glaucoma, 23(1), 23-32. http://doi.org/10.1097//JG.0b013e31825c10dc

Pesudovs, K., Patel, B., Bradbury, J. a, \& Elliott, D. B. (2002). Reading speed test for potential central vision measurement. Clinical \& Experimental Ophthalmology, 30 $183-186$. 
Rayner, K. (1998). Eye Movements in Reading and Information Processing : 20 Years of Research. Psycological Bulletin, 124(3), 372-422.

Rayner, K. (2009a). Eye Movements and Attention In Reading, Scene Perception, and Visual Search. Quarterly Journal of Experimental Psychology, 62(8), $1457-1506$.

Rayner, K. (2009b). Eye movements and attention in reading , scene perception , and visual search, 62(8), 1457-1506. http://doi.org/10.1080/17470210902816461

Rayner, K., \& Fischer, M. (1996). Mindless reading revisited: eye movements during reading and scanning are different. Perception \& Psychophysics, 58(5), $734-47$. http://doi.org/10.3758/BF03213106

Rosenfield, M., \& Cohen, A. S. (1996). Repeatability of clinical measurements of the amplitude of accommodation. Ophthalmic and Physiological Optics, 16(3), 247249. http://doi.org/10.1046/j.1475-1313.1996.95000933.x

Scheiman, M., Gallaway, M., Frantz, K. a, Peters, R. J., Hatch, S., Cuff, M., \& Mitchell, G. L. (2003). Nearpoint of convergence: test procedure, target selection, and normative data. Optometry and Vision Science, 80(3), 214-225. http://doi.org/10.1097/00006324-200303000-00011

Seiple, W., Rosen, R. B., \& Garcia, P. M. T. (2013). Abnormal Fixation in Individuals With Age-Related Macular Degeneration When Viewing an Image of a Face. Optometry \& Vision Science, 90(1), 1-12.

Sunness, J. S., Applegate, C. A., Haselwood, D., \& Rubin, G. S. (1996). Fixation Patterns and Reading Rates in Eyes with Central Scotomas from Advanced Atrophic Age-related Macular Degeneration and Stargardt Disease. Ophthalmology, 103(9), 1458-1466.

Sunness, J. S., Bressler, N. M., Tian, Y., Alexander, J., \& Applegate, C. A. (1999). Measuring Geographic Atrophy in Advanced Age-Related Macular Degeneration. Invest Ophthalmol Vis. Sci., 40(8), 1761-1769.

Varsori, M., Perez-Fornos, A., Safran, A. B., \& Whatham, A. R. (2004). Development of a viewing strategy during adaptation to an artificial central scotoma. Vision Research, 44(23), 2691-2705. http://doi.org/10.1016/j.visres.2004.05.027 